\title{
JÓZEF KWATERKO
}

Université de Varsovie

iD 0000-0002-0156-6423

\section{Carnavalisation et travail des stéréotypes dans La Guerre, yes sir! (1968) de Roch Carrier}

\author{
Carnavalization and Stereotyping \\ in La Guerre, yes sir! (1968) by Roch Carrier
}

\begin{abstract}
Carrier's novel was published in the same year as Nègres blancs d'Amérique by Pierre Vallières and it relies on the same dialectics between the colonizer and the colonized that was prevalent in Québécois narrative prose in the 1960s. While being grounded in this cultural context, Carrier transforms this dialectic by setting his narrative in a rural environment and against the background of World War II (the visitation of a fallen French-Canadian soldier whose remains are escorted to his native village by English-Canadian comrades). Such aesthetic reworking complexifies the ideological message of Carrier's novel. I will study the interplay between national ideology and Carrier's narratives strategies and particularly on the clash between ethnic stereotypes and the «carnavalesque» and the grotesque.
\end{abstract}

KeY Words: Québécois novel, ideology, stereotyping, carnavalization, ethnicity

La littérature est un des éléments constitutifs et expression privilégiée du discours social qui correspond, selon Marc Angenot, à «tout ce qui se dit et s'écrit dans un état de société (ANGENOT, 1989: 13). L'écrivain, dans cette perspective, prélève et «travaille» certains éléments du discours social à partir desquels il construit une œuvre qui, une fois produite, devient à son tour porteur du concert social global - avec tous les lieux communs de la doxa de son époque. La notion de «travail» du texte sur des représentations sociales telles qu'elles sont véhiculées par le discours social est surtout importante pour les approches sociocritiques. Il s'agit d'un rapport de médiation entre le texte et social, de la socialité du texte, telle que définie par Claude Duchet (Duchet, 1979: 4). La mise en texte du social, sa textualisation, ne recouvre pas les objets sociaux ou les signifiés historiques absolus, mais relève de cadres axiologiques, de conjonctures idéologiques et des schèmes discursifs inscrits dans les réseaux 
symboliques et dans l'imaginaire social, bref, dans toute cette matière verbale sur laquelle le texte est appelé à travailler. Comme le dit succinctement Régine Robin, le principal objet interrogé par la sociocritique «c'est le statut du social dans le texte et non le statut social du texte, c'est statut de l'historicité dans le texte et non le statut historique du texte» (RoBIN, 1993: 10).

Publié en 1968, la même année que le fameux Nègres blancs d'Amérique de Pierre Vallières, récit autobiographique d'un militant du Front de Libération du Québec auquel se greffent un essai historique, La Guerre, yes sir! de Roch Carrier est un roman fortement marqué par l'idéologie de la décolonisation, identifiable comme dispositif discursif dans plusieurs romans québécois de la Révolution tranquille (1960-1970) : p. ex : La nuit de Jacques Ferron, Prochain épisode (1965) d'Hubert Aquin, Le couteau sur la table (1965) de Jacques Godbout. Carrier met en fiction le rejet de l'enrôlement obligatoire des Québécois dans l'armée canadienne en 1942 après la déclaration de la guerre à l'Allemagne par gouvernement fédéral de Mackenzie King en septembre 1939. Le refus de la conscription des Québécois à ce conflit international est massif, malgré le recours à la propagande et l'instauration des mesures de guerre, tandis que les Canadiens anglais appuient majoritairement, la circonscription.

Le titre, La Guerre, yes sir !, bilingue, apparaît d'emblée comme une antiphrase; l'adhésion à la guerre ne peut s'énoncer qu'en anglais, tandis que la réplique "yes sir!» se donne pour une ruse, une obéissance feinte qui exprime en substance le refus du minoritaire à obtempérer aux ordres de l'autre, à l'imposition de son hégémonie. Si la signification et la teneur de cette transgression semblent décalées par rapport au contexte historique de la Deuxième Guerre mondiale, elles n'en sont pas moins importantes pour la scène discursive québécoise des années 1960 qui se nourrit de positions conflictuelles et d'idéologèmes, au sens que Marc Angenot assigne au dispositif polémique et dialogique (ANGENOT, 1989: 894), tels que «absence historique du Québec», et son contraire, «l'entrée du Québec dans l'Histoire». Comme le dit Élisabeth Nardout-Lafarge :

Lorsque paraît, en 1968, La Guerre? Yes sir! de Roch Carrier (sic !: le premier segment n'est pas suivi d'interrogation), la Deuxième Guerre Mondiale n'est plus un thème d'actualité, elle fait partie de l'histoire nationale. C'est dire qu'elle appartient désormais, pour reprendre la formulation de Angenot, à un autre "répertoire topique», partiellement déchargé de son potentiel conflictuel. Il n'en est pas mois significatif qu'un romancier choisisse, dans le patrimoine historique disponible, cet élément plutôt qu'un autre pour le soumettre au travail de la fiction.

NARdout-Lafarge, 1991: 56

En effet, le choix de Carrier de transposer dans son roman le conflit historique ancien pour en faire le lieu des affrontements socio-discursifs de son temps, là où s'exprime le nouveau «nous» québécois, au seuil de l'Histoire, est 
une entreprise complexe et ambiguë. Premier effet de brouillage du sens (d'opacification) : la guerre elle-même n'aura pas lieu. Elle reste mise à distance, évoquée en permanence comme «leur maudite guerre» (CARRIER, 1968: 10), celle qui a tué Corriveau, "un petit Canadien français, fils du village», une guerre «que les Anglais d'Angleterre, des États-Unis et du Canada avaient déclarée aux Allemands» (100). Cette guerre "anglaise» et lointaine où s'affrontent les « vieux pays» (11) et dont la présence est réduite à l'échelle locale, l'espace du village, couvert de neige, et au temps de la veillée funéraire, tourne tout entière autour de la mort de Corriveau dont le corps est rapatrié dans un cercueil escorté par six soldats britanniques qui vont se bagarrer avec les paysans venus à la maison des parents du défunt. Or, cette mort du soldat canadien-français ne nous est pas racontée véritablement qu'à l'avant-dernière page du récit. En effet, le sort qui lui est réservé relève du grotesque le plus pur où le tragique s'imbrique au dérisoire, car on apprend que Corriveau a sauté sur une mine en allant aux latrines le pantalon baissé. Cette scène liant la mort aux excréments n'est pas isolée.

Le roman de Carrier est tout entier fondé sur le réalisme grotesque où la gaieté est mêlée d'horreur et le voisinage du sérieux et du risible, du rire et de l'angoisse, débouchent sans cesse sur la représentation carnavalesque (au niveau des images) du conflit ethnique opposant les paysans canadiens-français et les soldats anglais. Ainsi lorsque Joseph, réfractaire à la conscription, se coupe la main, celle-ci, durcie par le froid, est utilisée comme une rondelle de hockey par les enfants du village. Le récit abonde en d'autres renversements carnavalesques: le cochon échaudé pour le repas de funérailles est comparé au Christ en croix (20); les prières subissent des déformations blasphématoires: «Marie pleine de grâce» devient « «Marie pleine et grasse» (49) comme les tourtières cuites au four par la mère de Corriveau; le drapeau Union Jack posé sur le cercueil sert de nappe sur laquelle on fait ripaille.

Carrier pratique le grossissement grotesque à l'endroit de la guerre en reprenant le cliché du soldat cocu qui, au retour du front, trouve sa femme avec un amant. Ainsi Amélie accepte de cacher le déserteur Arthur qui, très vite, prend la place de son mari Henri, alors à la guerre, et lorsque ce dernier revient du front, elle profite de deux hommes sous un même toit à tour de rôle et en s'en satisfait à peine: "C'est la guerre - dit-elle - Il faut quelqu'un pour la faire. Il faut des hommes à la guerre et des hommes à la maison» (55). Il en va de même de Bérubé, soldat canadien-français qui se bat pour les Anglais et qui a épousé Molly, une prostitué canadienne-anglaise parce que la peur de l'enfer l'empêche de lui faire l'amour. Mais il épouse du même coup une femme «damnée », débordant d'appétit sexuel, que les villageois convoitent et craignent à la fois: « elle apparût comme une autre incarnation du diable, dans cette maison où l'on veillait un homme de mort» (81). Molly se présente aux funérailles vêtue de sa robe de mariée et assiste à la longue veillée au corps en priant en anglais. 
Qui plus est, lorsque Molly et Bérubé sont accueillis par la mère Corriveau, c'est dans le lit du fils mort à la guerre qu'elle les installe et ils s'y adonneront à leurs violents ébats amoureux pendant que les soldats anglais et le paysans se disputent la dépouille mortelle et se bagarrent.

Or, dans La Guerre, yes sir!, tout ce puissant investissement carnavalesque et le recours au grotesque se heurtent à la programmation idéologique du récit fondée sur les stéréotypes ethniques: les soldats et les paysans se disputent la dépouille mortelle dans une lutte qui devient une «théâtralisation» de la guerre (Nardout-Lafarge, 1994: 57). La mise en scène de cette lutte repose tout entière sur la caricature qui exclue le principe carnavalesque de la suppression joyeuse des distances entre les hommes, formulé par Bakhtine (BAKHTINE, 1970 : 188). Chez Cartier, la vision ethnique est fondée sur des oppositions trop accusées qui abolissent la relativisation carnavalesque. La juxtaposition joue à plein : les soldats anglais, méprisants, froids, l'œil impassible, montent la garde autour $\mathrm{du}$ cercueil et refusent de manger les tourtières et boire du cidre que leur offrent les parents de Corriveau. Devant eux, les paysans frustres et bourrus, mais bons vivants, portés sur la bagatelle, aimant la fête, se conter des histoires grivoises, rire, manger, boire, mais aussi bons chrétiens craignant l'Eternel pour leurs pêchés:

Assis sur la table de la cuisine, ou appuyé contre un mur à cause de l'équilibre difficile, l'assiette dans une main, le verre de cidre dans l'autre, la graisse de tourtière dégoulinant sur le menton ou sur les joues, ou bien la tête échouée sur un tas de vaisselle graisseuse, ou bien soutenu par le montant de la porte ouverte sur la neige et le froid, essayant de vomir de vertige, ou bien les deux mains sur les fesses généreuses d'Antoinette ou bien, essayant de transpercer du regard la laine ajustée sur les seins de Philomène, l'on mangeait de la tourtière juteuse au salon, dans l'odeur des bougies qui allaient s'éteindre et l'on priait dans l'odeur lourde de la cuisine, l'odeur de la graisse à laquelle se mêlait celle de la sueur de ces hommes et de ses femmes. [...] Ces gens ne doutaient pas que leur prière serait comprise. Ils priaient avec toute leur force d'hommes, toute leur force de femmes accoucheuses d'enfants. Ils ne demandaient pas à Dieu que Corriveau revint sur terre; ils imploraient tout simplement Dieu de ne pas l'abandonner trop longtemps aux flemmes du purgatoire. Corriveau ne devait pas être en enfer. Il était un enfant du village, et il aurait semblé injuste, à ces villageois, qu'un de leurs enfants fût condamné aux flemmes éternelles.

$(65-66)$

Dans La Guerre, yes sir!, cette coprésence au niveau des images du profane et du sacré, du comique et du sérieux, qui est au cœur du carnavalesque populaire, telle que défini par Bakhtine, n'arrive pas à supprimer les stéréotypes qui polarisent le discours romanesque. Nous savons que le stéréotype est une représentation collective de schèmes culturels pour autant qu'il est figé, 
c'est-à-dire «connus d'avance» (Amossy, $1991:$ 22-23) et qu'il correspond à un modèle culturel daté (Amossy, Herschberg Pierrot, 1997: 64). En effet, chez Carrier, les différences culturelles où les types nationaux sont mis en épingle à partir d'un répertoire restreint de traits physiques et moraux, privés d'individualité, construisent plutôt ce que Marc Angenot appelle une «imagologie» (ANGENOT, 1989: 270-272) qui s'imprègne de l'imaginaire social et le façonne en retour. À ce niveau, comme le dit Elisabeth Nardout-Lafarge:

[...] le discours confirme, faisant écho au discours social, l'idée selon laquelle Canadiens et Européens n'ont pas vécu la même guerre, même si parfois ils ont combattu ensemble. La fiction actualise à sa manière le processus de différenciation qui s'opère alors dans le réel, tant par rapport à la France de l'origine, dont le souvenir n'est plus opérant, que par rapport à l'Angleterre, rejetée comme autorité légitime.

NARDOUT-LAFARGE, $1994: 58$

De manière significative, c'est sur plan de la narration que le récit de Carrier fait écho à ce que Marc Angenot qualifie de «gnoséologie romanesque» laquelle absorbe le doxa de son époque (ANGENot, 1989: 338). En effet, le roman «travaille» les discours social de son époque et la guerre qui a lieu dans la diégèse, ce n'est pas la lointaine guerre contre les Allemands, mais celle qui oppose le colonisateur anglais (l'« occupant») au colonisé québécois, le «nègre blanc d'Amérique ». En ce sens, le carnaval dans le roman, qui prend en charge l'Histoire, la grande, se trouve confronté à l'antithèse traditionnelle de l'histoire québécoise : celle du Canadien français et du Canadien anglais (NePveu, 1976: 57). Carier profite de cette antithèse et la met en fiction en vue réactualiser une blessure historique ancienne qui remonte à l'époque à de l'échec de la Révolte des Patriotes en 1838 contre le pouvoir britannique de la colonie. Cette révolte mort-née est à l'origine de la sanction proposée par Lord Durham, envoyé de Londres, qui dans son fameux Rapport de 1839 recommande au gouvernement britannique l'union du Bas Canada (majoritairement francophone) et du Haut Canada (majoritairement anglophone) en une seule province et d'assimiler les Canadiens qu'il traite de «peuple sans histoire et sans littérature» (Durham, 1990: 237). Dans son Rapport, Durham parle ainsi de sa stratégie d'assimilation:

On peut dire que si les Français ne sont pas une race aussi civilisée, aussi énergique, aussi apte à s'enrichir que celle qui les environne, ils sont par ailleurs un peuple aimable, vertueux et satisfait, possédant tout l'essentiel du confort matériel. [...] Si les querelles de deux races sont irréconciliables, on peut rétorquer que la justice exige la soumission de la minorité à la suprématie des anciens et plus nombreux occupants de la province, et non que la minorité prétende forcer la majorité à perdre ses institutions et ses coutumes. 
Tapie dans la mémoire collective québécoise, la maxime assimilatrice et l'invective historique de Durham («un peuple sans histoire et sans littérature») resurgit dans les années 1960 comme noyau conflictuel, figuré in absentia, de la majorité des enjeux discursifs (dans les essais, dans le discours indépendantiste ou autonomiste) aux prises avec la problématique décolonisatrice qui se rapporte à l'aliénation et de la dépossession identitaire des Québécois (voir KwATERKo, 1998: 40). Elle instaure en même temps un contre-discours, un vouloir-dire historique propre à un nouveau dispositif sémantique à valeur optative et prédicative - «nous aurons une histoire» - qui caractérise la modernité littéraire québécoise (visible surtout dans la poésie des poètes groupés autour des éditions de l'Hexagone, dite la poésie du pays). Comme le dit Pierre Nepveu:

Le «nouveau roman» québécois des années 1960 puise une bonne partie de son énergie dans cette manière d'«accuser» les traits de la dépossession, de faire de sa modernité un témoignage direct de l'aliénation québécoise, et de cette aliénation la forme même d'une écriture moderne, c'est-à-dire hyperbolique, tragi-comique, paradoxale.

Nepveu, 1988: 58

Il n'est pas étonnant que dans La Guerre yes sir! toute la dialectique textuelle entre identité-différence, entre la civilisation (la retenue et la discipline absolue des soldats anglais) et la barbarie (le déchaînement sauvage des villageois canadiens-français), se met au service d'une cause à défendre. Elle est particulièrement saillante à un point névralgique de l'intrigue, central pour l'investissement idéologique du roman, notamment lorsque les soldats anglais s'apprêtent à expulser les paysans de la maison des Corriveau - geste d'occupation symbolique de la maison paternelle et, pars pro toto, du pays. C'est là également que la résurrection textuelle du spectre de Durham et de sa glose sur l'an-historicité des Canadiens français va pouvoir apparaître comme une étrangeté radicale :

Les soldats $[\ldots]$ avaient regardé d'un œil impassible cette fête sauvage [...] mais le dégoût leur serrait les lèvres. Quelle sorte d'animaux étaient donc ces French Canadians? Ils avaient des manières de pourceaux dans la porcherie. [...] ils étaient des vrais porcs, ces French Canadians dont la civilisation consistait à boire, manger, péter, roter. Les soldats savaient depuis longtemps que les French Canadians étaient des porcs. «Donnez-leur à manger, donnez-leur où chier et nous aurons la paix dans le pays », disait-on. [...] Corriveau, ce French Canadian qui dormait sous leur drapeau, dans un uniforme semblable à celui dont ils étaient si orgueilleux , ce Corriveau était aussi un porc. [...] Le sergent dessina dans sa tête un plan d'occupation. Ses subalternes se souvenaient de ce qu'ils avaient appris à l'école. Les French Canadians étaient solitaires, craintifs, peu intelligents; ils n’étaient doués ni pour le gouvernement, ni pour le commerce, ni pour l'agriculture; mais ils faisaient beaucoup d'enfants. Quand les Anglais étaient arrivés dans la colonie, les French Canadians étaient moins 
civilisés que les Sauvages. Puisque la France les avaient abandonnés, pourquoi n'acceptaient-ils pas le privilège de devenir Anglais? L’Angleterre les aurait civilisés. Ils ne seraient plus des porcs de French Canadians. Ils sauraient comprendre une langue civilisée. Ils parleraient une langue civilisée, non un patois.

(90-92)

Cette représentation exacerbée et outrageusement dégradante des Canadiens français relève de la programmation idéologique du roman -, là où le «spectre » de Durham, la réappropriation discursive de la blessure historique ancienne, s'insinue dans le texte et demande «vengeance». C'est là aussi où la dialectique civilisation/barbarie qui sous-tend l'historicité du texte, nonobstant tous les renversements carnavalesques effectifs et toutes les images du rabaissement grotesque, se trouve en quelque sorte plaquée du dehors sur le texte. Car s'il est vrai que dans ce regard «anglais » sur l'identité culturelle québécoise l'effet de citation intertextuelle mobilise dans les années de la Révolution tranquille l'idéologème de l'aliénation historique évoqué précédemment - la Non-Histoire versus l'entrée dans l'Histoire -, son mode d'articulation est ici bien différent. En l'occurrence, l'interprétation du conflit ethnique par le personnage interposé - le soldat anglais - fait surgir un discours mental dont le caractère «étranger » et» hostile est si ostensible que tout dialogisme des points de vue et des discours (tel que l'entend Bakhtine) s'en trouve court-circuité. Et cela n'ont pas tant à cause de la perspective anglaise ou de la référence à des manuels d'histoire canadienne qui corroborent le propos dégradant de Durham (les manuels anglais des années 1950 s'y référent sans ambages). Ce qui importe ici, c'est l'intégration de cette perspective dans le discours narratif suivant le principe propre au récit à thèse, suivant lequel les commentaires interprétatifs du narrateur et du personnage sont redondants (Suleiman, 1983 : 208-215). En ce sens, le personnage du soldat anglais devient en quelque sorte le double inversé du narrateur québécois qui tend à démentir la prétendue sauvagerie des «French Canadians ».

Cette redondance est partout présente chez Carrier. On peut dire dès lors que La Guerre, yes sir! est un roman carnavalesque privé de carnavalisation et que malgré l'hétérophonie (la diversité de voix, en l'occurrence du registre sérieux et populaire) et l'hétéroglossie (diversité de langues, en l'occurrence l'alternance du français et de l'anglais), on y chercherait en vain une trace quelconque de l'hétérologie - cette diversité de discours indispensable, selon Bakhtine, à la polyphonie et au dialogisme romanesques (ToDorov, 1981 : 89).

$\mathrm{Vu}$ sous cet angle, le roman de Carrier pose avec acuité le problème de contraintes institutionnelles à l'usage du carnavalesque à des fins idéologiques dans nombre de romans québécois des années 1960. En désignant la littérature québécoise de cette période par la notion kafkaïenne de «littérature mineure» Régine Robin constate avec insistance : 
La visée du «mineur» a eu tendance à tout récupérer, aussi bien les systèmes métaphoriques que les allégories, les récits les plus éloignés en apparence du politique. Le mineur se réapproprie tout: le parodique, le carnavalesque, la langue, l'oralité du parlé dans une sur-conscience linguistique qui finit par jouer le rôle d'en enfermement identitaire et qui par là reproduit l'identité close, la petite communauté.

RoBIN, 1989: 175

C'est pourquoi, si la mise en texte de l'Histoire par Roch Carrier (i.e. la participation des Québécois à la Deuxième Guerre Mondiale) est une réponse fictionnelle à l'aliénation qui hante l'imaginaire social québécois des années 1960, elle signale en même temps une extraordinaire pression du discours social dans l'espace propre au littéraire.

\section{Bibliographie}

Amossy, Ruth 1991 : Les idées reçues. Sémiologie du stéréotype. Paris, Nathan.

Amossy, Ruth; Herschberg Pierrot, Anne 1997: Stéréotypes et clichés. Langue, discours, société. Paris, Nathan.

Angenot, Marc 1989: 1889. Un état du discours social. Longueil, Le Préambule.

BAKhtine, Mikhaïl 1970 : L'Euvre de François Rabelais et la culture populaire au Moyen Âge et sous la Renaissance. Traduit du russe par Andrée Robel. Paris, Gallimard.

CARrIER, Roch 1968: La guerre, yes sir!. Montréal, Éditions du Jour.

Duchet, Claude 1979: «Positions et perspectives». In: Claude Duchet (dir.): Sociocritique. Paris, Nathan, p. 3-8.

Durham, Lord John George Lambton 1990 : Le rapport Durham. Document. Montréal, L'Hexagone.

KWATERKo, Józef 1998: Le roman québécois et ses (inter)discours. Analyses sociocritiques. Québec, Nota bene.

Nardout-Lararge, Elisabeth 1991: «Stratégies d'une mise à distance: la Deuxième Guerre mondiale dans les textes québécois ». Études françaises, ${ }^{\circ} 2$, p. 43-60.

NePveu, Pierre 1976: «Le Grotesque dans La guerre, yes sir!». Nord, n 6, p. 4-59.

Nepveu, Pierre 1988: L'écologie du réel. Mort et naissance de la littérature québécoise contemporaine. Montréal, Boréal.

RoBin, Régine 1989: Le roman mémoriel: de l'histoire à l'écriture du hors-lieu. Longueil. Le Préambule.

Robin, Régine 1993 : «Pour une socio-poétique de l'imaginaire social». Discours social/Social Discourse, vol. 5, n 1-2, p. 7-32.

Suleiman, Susan Rubin 1983 : Le roman à these ou l'autorité fictive. Paris, Presses universitaires de France.

Todorov, Tzvetan 1981 : Mikhaïl Bakhtine le principe dialogique. Paris, Seuil. 


\section{Note bio-bibliographique}

Józef Kwaterko est professeur titulaire à l'Université de Varsovie où il dirige le Centre d'études en civilisation canadienne-française et en littérature québécoise. Il est auteur de Le roman québécois de 1960 à 1960: idéologie et représentation littéraire, 1989, French-Canadian and Québécois Novels, 1950-1990, 1996 (en collaboration avec Irène Geller et Jan Miernowski), Le roman québécois et ses (inter)discours. Analyses sociocritiques, 1998 et d'un ouvrage en polonais, Dialogi z Ameryka. O frankofońskiej literaturze w Québecu i na Karaibach (2003). Il a codirigé également deux ouvrages collectifs, publiés en 2006: L'humour et le rire dans les littératures francophones des Amériques et L'imaginaire du roman québécois (en collaboration avec Max Roy et Petr Kyloušek) ainsi que Kanade, Die Goldene Medine? Perspectives on Canadian Jewish Literature and Culture / Perspectives sur la littérature et culture juives canadiennes (Brill-Rodopi, 2018).

j.kwaterko@uw.edu.pl 